\title{
Perioperative Outcomes of Non-intubated Versus Intubated Video-assisted Thoracoscopic Surgery in Different Thoracic Procedures: a Propensity Score- matched Analysis
}

Chompunoot Pathonsamit

Navamindradhiraj University

Apichat Tantraworasin

Chiang Mai University

Sujaree Poopipatpab

Navamindradhiraj University

Sira Laohathai ( $\nabla$ sira_l@hotmail.com )

Navamindradhiraj University

\section{Research Article}

Keywords: Thoracic Surgery, Video-Assisted, Propensity Score, non-intubated

Posted Date: December 7th, 2021

DOI: https://doi.org/10.21203/rs.3.rs-1123170/v1

License: (c) (i) This work is licensed under a Creative Commons Attribution 4.0 International License.

Read Full License 


\section{Abstract}

Background: Non-intubated video-assisted thoracoscopic surgery (NIVATS) is increasingly performed in different types of thoracic procedures. Based on the anesthetic perspective, the outcomes of this method are limited. General anesthesia with intubation and controlled ventilation for video-assisted thoracoscopic surgery (IVATS) is a standard technique. The current study aimed to compare the pulmonary gas exchange between NIVATS and IVATS, with a focus on desaturation event.

Methods: This was a retrospective study conducted at Vajira Hospital. Data were collected from the hospital medical record database between January 9, 2019, and May 15, 2020. A propensity scorematched analysis was used to adjust the confounders by indications and contraindication between NIVATS and IVATS. The perioperative outcomes of VATS and NIVATS were compared by the regression analysis method.

Results: In total, 180 patients were included in the analysis. There were 98 and 82 patients in the NIVATS and IVATS groups, respectively. After a propensity score matching, the number of patients with similar characteristics decreased to 52 per group. None of the patients in both groups experienced desaturation. The lowest oxygen saturation of the NIVATS and IVATS groups did not significantly differ $(96.5 \%$ vs. $99 \%$, respectively; $p=0.185$ ). The NIVATS group had a significantly higher ETco2 peak than the IVATS group (43 vs. $36 \mathrm{mmHg}$, respectively; $p<0.001$ ). According to the regression analysis, the NIVATS group had a significantly shorter anesthetic induction time (Mean difference $(M D)=-5.135 \mathrm{~min}(95 \% \mathrm{Cl}=(-8.878)$ $(-1.391))$ and lower volume of blood loss $(\mathrm{MD}=-75.565 \mathrm{ml}(95 \% \mathrm{Cl}=(-131.08)-(-20.65)$ but a higher intraoperative $\mathrm{ETCO}_{2}$ than the IVATS group (MD $=4.561 \mathrm{mmHg}(95 \% \mathrm{Cl}=1.852-7.269)$. Four patients in the NIVATS group required conversion to intubation due to difficulties encountered when using the surgical technique $(7.7 \%, p=0.041)$. Seven patients in the IVATS group, but none in the NIVATS group, presented with sore throat $(13.5 \%$ vs. $0 \%$, respectively; $p=0.006)$. Moreover, none of the patients in both groups experienced postoperative pneumonia, underwent reoperation, or died.

Conclusions: The anesthetic and surgical outcomes of NIVATS were comparable to those of IVATS.

\section{Background}

General anesthesia with intubation and controlled ventilation for video-assisted thoracoscopic surgery (IVATS) is a standard technique. Several lung isolation devices such as double-lumen endobronchial tube (DLT), bronchial blocker, and single-lumen tube were applied to achieve a good surgical exposure. However, previous reports showed that the occurrence of complications correlated with intubation, which range from tracheal rupture ${ }^{(1-3)}$ and dental damage ${ }^{(4)}$ to sore throat. Moreover, ventilator-induced lung injury after one-lung ventilation (OLV) was reported in some studies ${ }^{(5)}$.

Non-intubated video-assisted thoracoscopic surgery (NIVATS) is an alternative technique that has been increasingly performed during the last two decades. This technique is implemented for different types of 
thoracic procedures, such as wedge resection, segmentectomy, lobectomy, lung volume reduction, and mediastinal tumor surgery, with successful surgical outcomes ${ }^{(6-11)}$.

According to the anesthetic perspective, studies about NIVATS technique reported in oxygenation and ventilation during procedure, complications correlated with anesthesia, and anesthetic and recovery time are limited. Some reports have shown that pulmonary gas exchange is preserved during the procedure. However, data about anesthetic-related complications are still lacking ${ }^{(12)}$.

Therefore, the current study aimed to compare the pulmonary gas exchange between NIVATS and IVATS, with a focus on desaturation event, which was defined as an oxygen saturation (SpO2) of $<90 \%$ assessed using a pulse oximetry. The other outcomes were anesthetic induction and surgical time, conversion rate to IVATS, anesthetic and surgical complications, volume of blood loss, length of hospital stay, and 30-day mortality rate.

\section{Methods}

This study was approved by the Vajira ethics committee (COA 103/63), and it was registered in the Thai Clinical Trials Registry (TCTR20201027004). The need for informed consent was waived due to the retrospective nature of the study.

\section{Study design and patients}

In total, 180 VATS procedures in Vajira Hospital, Navamindradhiraj University, Thailand, were retrospectively analyzed. Data were collected from the hospital medical record database between January 9, 2019, and May 15, 2020.

The inclusion criteria were as follows: patients who were scheduled to undergo VATS for pulmonary resection and who were aged $\geq 18$ years old with an American Society of Anesthesiologist physical status of I-III. The exclusion criteria were as follows: patients who require reoperation, emergency surgery, and conversion to open thoracotomy.

\section{Sample size and statistical analysis}

The sample size was calculated assuming that there was no difference in desaturation events between the two techniques. A previous study showed that $4 \%$ of desaturation events occurred in IVATS. ${ }^{(13)}$ Approximately 48 patients were required per group, with an alpha value of 0.05 and beta value of 0.2 . For data completion, 180 patients were included in the study.

All statistical analyses were conducted using Stata Statistical Software version SE 13 (StataCorp LP, College Station, TX, the USA). Categorical variables were presented as frequencies and proportions and continuous variables as median $\left(P_{25}-P_{75}\right)$. A 1:1 propensity score-matched analysis was performed to reduce differences and selection bias between the NIVATS and IVATS groups. The variables included in the propensity score-matched model were age, sex, body mass index, smoking status, underlying disease, 
diagnosis, and thoracic procedure. All variables between the two groups had a standardized difference. The chi-square test was used to compare categorical variables and differences between the two groups. The Mann-Whitney $U$ test was applied to assess continuous data and skewed distributed data. A linear regression analysis was performed to assess the associations between the anesthetic technique and anesthetic induction time, highest intraoperative end-tidal carbon dioxide (ETcO2), volume of blood loss, and length of hospital stay. A $p$-value of $<0.05$ indicated a statistically significant difference.

\section{Anesthetic technique}

All patients received premedication including paracetamol $500 \mathrm{mg}$ and gabapentin $300 \mathrm{mg} 2 \mathrm{~h}$ before surgery. Arterial line and Foley catheter were used in complicated surgeries alone. Epidural or paravertebral blockade was not used in any cases.

\section{NIVATS group}

The anesthetic technique used was based on the targeted controlled infusion of propofol and titration of fentanyl as total intravenous anesthesia. The depth of anesthesia was monitored using bispectral index to maintain the value between 40 and 60 . Oxygen was supplemented via facemask with a reservoir bag with an oxygen flow of $10 \mathrm{~L}$ per min. Spontaneous ventilation was maintained during the procedures. Lung collapse was achieved via iatrogenic pneumothorax. At the end of surgery, lung re-expansion was performed by applying positive pressure via mask ventilation, and intravenous drugs were discontinued. Patients were transferred to the post-anesthetic care unit and then to the ward or intensive care unit according to their conditions.

\section{IVATS group}

A double-lumen endobronchial tube (Broncho Cath DLT, Mallinckrodt Medical, Inc. St. Louis, MO) was inserted after induction with propofol, fentanyl, and cisatracurium. We selected a tube size of 32- or 35-Fr in women and 37- or 39-Fr in men. The proper tube position was confirmed based on breath sound and chest movement. Neuromuscular blockade was added according to schedule. Anesthesia was maintained by adjusting sevoflurane concentration and fentanyl titration properly according to hemodynamic changes. The protective ventilation strategy was used with a tidal volume of $4-5 \mathrm{~mL} / \mathrm{kg}$ and a positive end-expiratory pressure of $5 \mathrm{~cm} \mathrm{H}_{2} \mathrm{O}$, with a peak pressure of $<30 \mathrm{~cm} \mathrm{H}_{2} \mathrm{O}$. A fractional inspired oxygen tension of 1.0 was applied during OLV. Lung collapse was achieved via DLT clamping. At the end of surgery. lung re-expansion was conducted via positive pressure ventilation using the tube. Patients were extubated at the end of surgery based on the criteria. Patients were transferred to the postanesthetic care unit and then to the ward or intensive care unit according to their conditions.

\section{Surgical technique}

The NIVATS and IVATS groups underwent surgery performed by a single thoracic surgeon. At the beginning of surgery, all patients received local infiltration and 3th -8 th intercostal nerve blockade with $2 \%$ xylocaine with $10 \mathrm{~mL}$ of adrenaline $(1: 200,000)$ combined with $20 \mathrm{~mL}$ of $0.25 \%$ bupivacaine. Vagal nerve blockade, which aims to suppress cough reflex, was facilitated in the NIVATS group along using the 
same analgesic mixture. Patients routinely undergo chest tube placement at the end of surgery. In cautiously selected cases, tubeless surgery was considered based on the surgical condition of patients.

\section{Results}

In total, 314 patients were included in the study. Among them, 134 were excluded, and 180 patients were finally included in the analysis. There were 98 and 82 patients in the NIVATS and IVATS groups, respectively. The two groups differed in terms of baseline characteristics, diagnosis, and procedure. The NIVATS group was younger and had a lower incidence of comorbidities than the IVATS group. Moreover, the NIVATS group frequently diagnosed with benign lesion and underwent wedge resection. After a propensity score matching, both groups were found to have similar characteristics including age, body mass index, sex, smoking status, comorbidities, diagnosis, and thoracic procedure. The number of patients decreased to 52 per group (Table 1). 
Table 1

Patient characteristics before and after propensity score matching according to anesthesia technique.

\begin{tabular}{|c|c|c|c|c|c|c|c|c|}
\hline \multirow[t]{3}{*}{ Variables } & \multicolumn{4}{|c|}{ Before Propensity Score Matching } & \multicolumn{4}{|c|}{ After Propensity Score Matching } \\
\hline & \multicolumn{4}{|c|}{ (Full Patient Cohort) } & \multicolumn{4}{|c|}{$\begin{array}{l}\text { (Propensity Score Matched Patient } \\
\text { Cohort) }\end{array}$} \\
\hline & $\begin{array}{l}\text { NIVATS } \\
(n=98)\end{array}$ & $\begin{array}{l}\text { IVATS } \\
(n=82)\end{array}$ & $\begin{array}{l}\mathrm{p}- \\
\text { value }\end{array}$ & STD & $\begin{array}{l}\text { NIVATS } \\
(n=52)\end{array}$ & $\begin{array}{l}\text { IVATS } \\
(n=52)\end{array}$ & $\begin{array}{l}\mathrm{p}- \\
\text { value }\end{array}$ & STD \\
\hline $\begin{array}{l}\text { Age (years); } \\
\text { median }\left(P_{25}-\right. \\
\left.P_{75}\right)\end{array}$ & $\begin{array}{l}54(38.8 \\
-65)\end{array}$ & $\begin{array}{l}60(44.8 \\
-69.3)\end{array}$ & $0.034^{\star}$ & -0.309 & $\begin{array}{l}55 \\
(43.5- \\
65.5)\end{array}$ & $\begin{array}{l}59.5 \\
(40.5- \\
68)\end{array}$ & 0.492 & -0.129 \\
\hline Gender; n (\%) & & & 0.663 & 0.065 & & & 0.693 & 0.078 \\
\hline Male & $\begin{array}{l}47 \\
(48 \%)\end{array}$ & $\begin{array}{l}42 \\
(51.2 \%)\end{array}$ & & & $\begin{array}{l}22 \\
(42.3 \%)\end{array}$ & $\begin{array}{l}24 \\
(46.2 \%)\end{array}$ & & \\
\hline Female & $\begin{array}{l}51 \\
(52 \%)\end{array}$ & $\begin{array}{l}40 \\
(48.8 \%)\end{array}$ & & & $\begin{array}{l}30 \\
(57.7 \%)\end{array}$ & $\begin{array}{l}28 \\
(53.9 \%)\end{array}$ & & \\
\hline $\begin{array}{l}\mathrm{BMI}\left(\mathrm{kg} / \mathrm{m}^{2}\right) \\
\text { median }\left(\mathrm{P}_{25}{ }^{-}\right. \\
\left.\mathrm{P}_{75}\right)\end{array}$ & $\begin{array}{l}22.1 \\
(19.6- \\
24.7)\end{array}$ & $\begin{array}{l}22.8 \\
(20.2- \\
26.7)\end{array}$ & 0.179 & -0.212 & $\begin{array}{l}22.6 \\
(19.8- \\
24.9)\end{array}$ & $\begin{array}{l}22.2 \\
(18.7- \\
26.5)\end{array}$ & 0.818 & 0.024 \\
\hline $\begin{array}{l}\text { Smoking status; } \\
\text { n (\%) }\end{array}$ & & & 0.721 & 0.053 & & & 0.426 & 0.156 \\
\hline Non-smoker & $\begin{array}{l}78 \\
(79.6 \%)\end{array}$ & $\begin{array}{l}67 \\
(81.7 \%)\end{array}$ & & & $\begin{array}{l}45 \\
(86.5 \%)\end{array}$ & $\begin{array}{l}42 \\
(80.8 \%)\end{array}$ & & \\
\hline Smoker & $\begin{array}{l}20 \\
(20.4 \%)\end{array}$ & $\begin{array}{l}15 \\
(18.3 \%)\end{array}$ & & & $\begin{array}{l}7 \\
(13.5 \%)\end{array}$ & $\begin{array}{l}10 \\
(19.2 \%)\end{array}$ & & \\
\hline \multicolumn{9}{|l|}{$\begin{array}{l}\text { Underlying } \\
\text { disease; n (\%) }\end{array}$} \\
\hline $\begin{array}{l}\text { Diabetes } \\
\text { mellitus }\end{array}$ & $\begin{array}{l}22 \\
(22.4 \%)\end{array}$ & $\begin{array}{l}19 \\
(23.2 \%)\end{array}$ & 0.908 & -0.017 & $\begin{array}{l}13 \\
(25 \%)\end{array}$ & $\begin{array}{l}11 \\
(21.2 \%)\end{array}$ & 0.642 & 0.039 \\
\hline Hypertension & $\begin{array}{l}30 \\
(30.6 \%)\end{array}$ & $\begin{array}{l}39 \\
(47.6 \%)\end{array}$ & $0.020^{*}$ & -0.351 & $\begin{array}{l}20 \\
(38.5 \%)\end{array}$ & $\begin{array}{l}21 \\
(40.4 \%)\end{array}$ & 0.841 & 0.000 \\
\hline Dyslipidemia & $\begin{array}{l}21 \\
(21.4 \%)\end{array}$ & $\begin{array}{l}28 \\
(34.1 \%)\end{array}$ & 0.056 & -0.285 & $\begin{array}{l}12 \\
(23.1 \%)\end{array}$ & $\begin{array}{l}12 \\
(23.1 \%)\end{array}$ & 1.000 & 0.000 \\
\hline $\begin{array}{l}\text { Cardiovascular } \\
\text { disease }\end{array}$ & $0(0 \%)$ & $5(6.1 \%)$ & $0.018^{*}$ & -0.358 & $0(0 \%)$ & $0(0 \%)$ & & \\
\hline $\begin{array}{l}\text { Cerebrovascular } \\
\text { disease }\end{array}$ & $1(1 \%)$ & $5(6.1 \%)$ & 0.094 & -0.275 & $\begin{array}{l}1 \\
(1.9 \%)\end{array}$ & $\begin{array}{l}1 \\
(1.9 \%)\end{array}$ & 1.000 & 0.000 \\
\hline COPD & $3(3.1 \%)$ & $9(11 \%)$ & $0.040^{\star}$ & -0.312 & $\begin{array}{l}3 \\
(5.8 \%)\end{array}$ & $\begin{array}{l}2 \\
(3.9 \%)\end{array}$ & 0.647 & 0.090 \\
\hline
\end{tabular}




\begin{tabular}{|c|c|c|c|c|c|c|c|c|}
\hline \multirow[t]{3}{*}{ Variables } & \multicolumn{4}{|c|}{ Before Propensity Score Matching } & \multicolumn{4}{|c|}{ After Propensity Score Matching } \\
\hline & \multicolumn{4}{|c|}{ (Full Patient Cohort) } & \multicolumn{4}{|c|}{$\begin{array}{l}\text { (Propensity Score Matched Patient } \\
\text { Cohort) }\end{array}$} \\
\hline & $\begin{array}{l}\text { NIVATS } \\
(n=98)\end{array}$ & $\begin{array}{l}\text { IVATS } \\
(n=82)\end{array}$ & $\begin{array}{l}\mathrm{p}- \\
\text { value }\end{array}$ & STD & $\begin{array}{l}\text { NIVATS } \\
(n=52)\end{array}$ & $\begin{array}{l}\text { IVATS } \\
(n=52)\end{array}$ & $\begin{array}{l}\mathrm{p}- \\
\text { value }\end{array}$ & STD \\
\hline Diagnosis; n (\%) & & & $0.022^{\star}$ & 0.349 & & & & \\
\hline Malignant & $\begin{array}{l}61 \\
(62.2 \%)\end{array}$ & $\begin{array}{l}64 \\
(78.1 \%)\end{array}$ & & & $\begin{array}{l}36 \\
(69.2 \%)\end{array}$ & $\begin{array}{l}37 \\
(71.2 \%)\end{array}$ & 0.830 & 0.042 \\
\hline Benign & $\begin{array}{l}37 \\
(37.7 \%)\end{array}$ & $\begin{array}{l}18 \\
(21.9 \%)\end{array}$ & & & $\begin{array}{l}16 \\
(30.8 \%)\end{array}$ & $\begin{array}{l}15 \\
(28.9 \%)\end{array}$ & 0.596 & \\
\hline Procedure; n (\%) & & & $0.000 *$ & 0.600 & & & 0.596 & 0.200 \\
\hline Wedge & $\begin{array}{l}78 \\
(79.6 \%)\end{array}$ & $\begin{array}{l}43 \\
(52.4 \%)\end{array}$ & & & $\begin{array}{l}37 \\
(71.2 \%)\end{array}$ & $\begin{array}{l}37 \\
(71.2 \%)\end{array}$ & & \\
\hline Segmentectomy & $2(2 \%)$ & $5(6.1 \%)$ & & & $\begin{array}{l}1 \\
(1.9 \%)\end{array}$ & $0(0 \%)$ & & \\
\hline Lobectomy & $\begin{array}{l}18 \\
(18.4 \%)\end{array}$ & $\begin{array}{l}34 \\
(41.5 \%)\end{array}$ & & & $\begin{array}{l}14 \\
(27 \%)\end{array}$ & $\begin{array}{l}15 \\
(27.9 \%)\end{array}$ & & \\
\hline
\end{tabular}

Abbreviation: STD, standardized difference; NIVATS, Non-intubated video-assisted thoracoscopic surgery; IVATS, General anesthesia with intubation and controlled ventilation for video-assisted thoracoscopic surgery; BMI, body mass index; COPD, chronic obstructive pulmonary disease.

In terms of anesthetic outcomes, both groups did not present with desaturation. The lowest SpO2 between the two groups did not significantly differ $(96.5 \%$ vs. $99 \%, p=0.185)$. By contrast, the NIVATS group had a significantly higher ETco2 peak than the IVATS group (43 vs. $36 \mathrm{mmHg}, p<0.001$ ) (Table 2). The ETco2 of NIVATS group was significantly higher than that of IVATS group.. The anesthetic induction time of the NIVATS group was significantly shorter than that of the IVATS group (10 vs. $15 \mathrm{~min}, p=0.005$ ) (Table 2). There was no difference in the overall anesthetic induction and operative time between the two groups. 
Table 2

Peri-operative outcomes between two anesthesia technique.

\begin{tabular}{|c|c|c|c|c|c|c|c|c|}
\hline \multirow[t]{3}{*}{ Variables } & \multicolumn{4}{|c|}{ Before Propensity Score Matching } & \multicolumn{4}{|c|}{ After Propensity Score Matching } \\
\hline & \multicolumn{4}{|c|}{ (Full Patient Cohort) } & \multicolumn{4}{|c|}{$\begin{array}{l}\text { (Propensity Score Matched Patient } \\
\text { Cohort) }\end{array}$} \\
\hline & $\begin{array}{l}\text { NIVATS } \\
(n=98)\end{array}$ & $\begin{array}{l}\text { IVATS } \\
(n=82)\end{array}$ & $\begin{array}{l}\mathrm{p}- \\
\text { value }\end{array}$ & STD & $\begin{array}{l}\text { NIVATS } \\
(n=52)\end{array}$ & $\begin{array}{l}\text { IVATS } \\
(n=52)\end{array}$ & $\begin{array}{l}\mathrm{p}- \\
\text { value }\end{array}$ & STD \\
\hline $\begin{array}{l}\text { Anesthetic } \\
\text { induction time } \\
\text { (min); } \\
\text { median }\left(\mathrm{P}_{25}-\right. \\
\left.\mathrm{P}_{75}\right)\end{array}$ & $\begin{array}{l}10(5- \\
15)\end{array}$ & $\begin{array}{l}13.5 \\
(10- \\
20)\end{array}$ & $0.009 *$ & -0.318 & $\begin{array}{l}10(5- \\
15)\end{array}$ & $\begin{array}{l}15(10- \\
20)\end{array}$ & $0.005^{\star}$ & -0.534 \\
\hline $\begin{array}{l}\text { Overall } \\
\text { anesthetic time } \\
\text { (min); } \\
\text { median }\left(\mathrm{P}_{25}-\right. \\
\left.\mathrm{P}_{75}\right)\end{array}$ & $\begin{array}{l}125(90 \\
-201.3)\end{array}$ & $\begin{array}{l}122.5 \\
(95- \\
171.3)\end{array}$ & 0.725 & 0.083 & $\begin{array}{l}145 \\
(97.5- \\
210)\end{array}$ & $\begin{array}{l}127.5 \\
(105- \\
175)\end{array}$ & 0.651 & 0.121 \\
\hline $\begin{array}{l}\text { Operative time } \\
\text { (min); } \\
\text { median }\left(P_{25}-\right. \\
\left.P_{75}\right)\end{array}$ & $\begin{array}{l}60(40- \\
95)\end{array}$ & $\begin{array}{l}112.5 \\
(63.8- \\
171.3)\end{array}$ & $0.000 *$ & -0.701 & $\begin{array}{l}67.5 \\
(40- \\
107.5)\end{array}$ & $\begin{array}{l}80(55- \\
167.5)\end{array}$ & 0.059 & -0.368 \\
\hline \multicolumn{9}{|l|}{$\begin{array}{l}\text { Anesthetic } \\
\text { results }\end{array}$} \\
\hline $\begin{array}{l}\text { Prevalence of } \\
\text { desaturation }\end{array}$ & $0(0 \%)$ & $0(0 \%)$ & & & $0(0 \%)$ & $0(0 \%)$ & & \\
\hline $\begin{array}{l}\text { The lowest } \\
\text { intraoperative } \\
\mathrm{SpO}_{2}(\%) \text {; median } \\
\left(\mathrm{P}_{25}-\mathrm{P}_{75}\right)\end{array}$ & $\begin{array}{l}100 \\
(96.8- \\
100)\end{array}$ & $\begin{array}{l}99.5 \\
(95.8- \\
100)\end{array}$ & 0.056 & 0.097 & $\begin{array}{l}96.5 \\
(92- \\
100)\end{array}$ & $\begin{array}{l}99(97- \\
100)\end{array}$ & 0.185 & -0.056 \\
\hline $\begin{array}{l}\text { The highest } \\
\text { intraoperative } \\
\mathrm{ETCO}_{2}(\mathrm{mmHg}) \\
\text { median }\left(\mathrm{P}_{25^{-}}\right. \\
\left.\mathrm{P}_{75}\right)\end{array}$ & $\begin{array}{l}44(40- \\
48)\end{array}$ & $\begin{array}{l}36.5 \\
(34- \\
40)\end{array}$ & $0.000 *$ & 0.837 & $\begin{array}{l}43 \\
(40.5- \\
48)\end{array}$ & $\begin{array}{l}36(34- \\
41)\end{array}$ & $0.000^{\star}$ & 0.655 \\
\hline \multicolumn{9}{|l|}{ Surgical results } \\
\hline $\begin{array}{l}\text { Blood loss }(\mathrm{ml}) ; \\
\text { median }\left(\mathrm{P}_{25^{-}}\right. \\
\left.\mathrm{P}_{75}\right)\end{array}$ & $\begin{array}{l}20(10- \\
50)\end{array}$ & $\begin{array}{l}50(10- \\
150)\end{array}$ & $0.000 *$ & -0.531 & $\begin{array}{l}20(10- \\
50)\end{array}$ & $\begin{array}{l}50(10- \\
125)\end{array}$ & $0.008^{*}$ & -0.534 \\
\hline
\end{tabular}




\begin{tabular}{|c|c|c|c|c|c|c|c|c|}
\hline \multirow[t]{3}{*}{ Variables } & \multicolumn{4}{|c|}{ Before Propensity Score Matching } & \multicolumn{4}{|c|}{ After Propensity Score Matching } \\
\hline & \multicolumn{4}{|c|}{ (Full Patient Cohort) } & \multicolumn{4}{|c|}{$\begin{array}{l}\text { (Propensity Score Matched Patient } \\
\text { Cohort) }\end{array}$} \\
\hline & $\begin{array}{l}\text { NIVATS } \\
(n=98)\end{array}$ & $\begin{array}{l}\text { IVATS } \\
(n=82)\end{array}$ & $\begin{array}{l}\mathrm{p}- \\
\text { value }\end{array}$ & STD & $\begin{array}{l}\text { NIVATS } \\
(n=52)\end{array}$ & $\begin{array}{l}\text { IVATS } \\
(n=52)\end{array}$ & $\begin{array}{l}\mathrm{p}- \\
\text { value }\end{array}$ & STD \\
\hline $\begin{array}{l}\text { Blood } \\
\text { transfusion; n (\%) }\end{array}$ & $1(1 \%)$ & $\begin{array}{l}4 \\
(4.9 \%)\end{array}$ & 0.179 & 0.23 & $0(0 \%)$ & $\begin{array}{l}3 \\
(5.8 \%)\end{array}$ & 0.079 & 0.350 \\
\hline $\begin{array}{l}\text { Tubeless surgery; } \\
\text { median }\left(\mathrm{P}_{25^{-}}\right. \\
\left.\mathrm{P}_{75}\right)\end{array}$ & $3(3-5)$ & $\begin{array}{l}5(4- \\
8.3)\end{array}$ & $0.000 *$ & -0.543 & $3(3-4)$ & $5(4-7)$ & $<.001 *$ & -0.408 \\
\hline \multicolumn{9}{|l|}{ Complications } \\
\hline $\begin{array}{l}\text { Conversion to } \\
\text { GAVATS; } \mathrm{n}(\%)\end{array}$ & $\begin{array}{l}6 \\
(6.1 \%)\end{array}$ & $0(0 \%)$ & $0.032^{*}$ & 0.361 & $\begin{array}{l}4 \\
(7.7 \%)\end{array}$ & $0(0 \%)$ & $0.041^{*}$ & 0.408 \\
\hline Sore throat; n (\%) & $0(0 \%)$ & $\begin{array}{l}10 \\
(12.2 \%)\end{array}$ & $0.000^{\star}$ & 0.527 & $0(0 \%)$ & $\begin{array}{l}7 \\
(13.5 \%)\end{array}$ & $0.006^{*}$ & 0.558 \\
\hline $\begin{array}{l}\text { Other anesthetic } \\
\text { complication; } \mathrm{n} \\
(\%)\end{array}$ & $\begin{array}{l}4 \\
(4.1 \%)\end{array}$ & $0(0 \%)$ & 0.127 & 0.292 & $\begin{array}{l}2 \\
(3.9 \%)\end{array}$ & $0(0 \%)$ & 0.153 & 0.283 \\
\hline $\begin{array}{l}\text { Reoperation; n } \\
\text { (\%) }\end{array}$ & $2(2 \%)$ & $\begin{array}{l}1 \\
(1.2 \%)\end{array}$ & 1.000 & 0.065 & $0(0 \%)$ & $0(0 \%)$ & & \\
\hline Pneumonia; n (\%) & $0(0 \%)$ & $0(0 \%)$ & & & $0(0 \%)$ & $0(0 \%)$ & & \\
\hline $\begin{array}{l}\text { Chest radiograph } \\
\text { results; } n(\%)\end{array}$ & & & 0.749 & 0.047 & & & 0.169 & 0.270 \\
\hline Normal & $\begin{array}{l}92 \\
(93.9 \%)\end{array}$ & $\begin{array}{l}76 \\
(92.7 \%)\end{array}$ & & & $\begin{array}{l}51 \\
(98.1 \%)\end{array}$ & $\begin{array}{l}48 \\
(92.3 \%)\end{array}$ & & \\
\hline Atelectasis & $\begin{array}{l}6 \\
(6.1 \%)\end{array}$ & $\begin{array}{l}6 \\
(6.1 \%)\end{array}$ & & & $\begin{array}{l}1 \\
(1.92 \%)\end{array}$ & $\begin{array}{l}4 \\
(7.7 \%)\end{array}$ & & \\
\hline $\begin{array}{l}\text { Hospital stays } \\
\text { (day); median } \\
\left(\mathrm{P}_{25}-\mathrm{P}_{75}\right)\end{array}$ & $\begin{array}{l}5(4- \\
7.3)\end{array}$ & $\begin{array}{l}7(5- \\
11)\end{array}$ & $0.000^{*}$ & -0.426 & $5(4-7)$ & $\begin{array}{l}6(5- \\
10)\end{array}$ & $0.004^{*}$ & -0.341 \\
\hline Mortality; n (\%) & $0(0 \%)$ & $0(0 \%)$ & & & $0(0 \%)$ & $0(0 \%)$ & & \\
\hline
\end{tabular}

Abbreviation: STD, standardized difference; NIVATS, Non-intubated video-assisted thoracoscopic surgery; IVATS, General anesthesia with intubation and controlled ventilation for video-assisted thoracoscopic surgery; $\mathrm{SpO}_{2}$, oxygen saturation; $\mathrm{ETCO}_{2}$, end-tidal carbon dioxide.

In terms of surgical outcomes, the NIVATS group had a significantly lower volume of blood loss and a shorter length of hospital stay than the IVATS group ( $20 \mathrm{vs.} 50 \mathrm{~mL}, p=0.008$ and 5 vs. 6 days, $p=0.004)$. There was no mortality in both groups. 
In the regression analysis, the NIVATS group had a significantly shorter anesthetic induction time and lower volume of blood loss but a higher intraoperative $\mathrm{ETCO}_{2}$ than the IVATS group (Table 3 ).

Table 3

Multiple linear regression analysis of each outcome variable comparing between NIVATS and IVATS

\begin{tabular}{|llll|}
\hline Outcome variables & Mean difference & $95 \% \mathrm{Cl}$ & $\mathbf{p}$-value \\
\hline Anesthetic induction time $(\mathrm{min}) ;$ & -5.135 & $(-8.878)-(-1.391)$ & $0.008^{\star \mathrm{a}}$ \\
\hline The highest intraoperative ETCO $2(\mathrm{mmHg}) ;$ & 4.561 & $1.852-7.269$ & $0.001^{\star b}$ \\
\hline Estimate blood loss $(\mathrm{ml}) ;$ & -75.565 & $(-131.08)-(-20.65)$ & $0.008^{\star \mathrm{c}}$ \\
\hline Hospital stays (day); & -1.923 & $(-3.912)-0.027$ & $0.053^{\mathrm{d}}$ \\
\hline
\end{tabular}

Notes: Analyzed by Multiple Linear Regression model

${ }^{a}$ Adjusted by Age, BMl, Gender, Smoking status and Diagnosis.

${ }^{\text {b }}$ Adjusted by Age, Gender, DM, HT, Stroke and operation time.

${ }^{c}$ Adjusted by Age, Gender, BMI and Procedure.

d Adjusted by Age, Gender, Procedure, Conversion to GAVATS, Sore throat and Other anesthetic complication.

In terms of postoperative complications, four patients in the NIVATS group required conversion to intubation with controlled ventilation during surgery $(7.7 \%, p=0.041)$. The reasons for conversion were difficulties using the surgical technique due to factors such as severe calcified lymph node and bleeding. In total, seven patients in the IVATS group, but none in the NIVATS group, experienced sore throat $(13.5 \%$ vs. $0 \%, p=0.006$ ). There was no difference in terms of other anesthetic complications between the two groups. None of the patients had postoperative pneumonia or reoperation. There was no difference in terms of atelectasis on postoperative chest radiography between the two groups.

\section{Discussion}

Based on the anesthetic perspective, hypoxia and hypercapnia are the most common issues during OLV. Intubation with controlled ventilation is an essential strategy. Hence, this study aimed to compare the pulmonary gas exchange and perioperative conditions between NIVATS and IVATS for thoracic surgery. Results showed that the NIVATS and IVATS groups did not experience desaturation. In previous studies, the incidence of desaturation during OLV via the tube was between $4 \%$ and $20 \%(13-15)$.

The high incidence rate might be attributed to different factors, including varying characteristics of patients, anesthetic technique and expertise, surgical technique, and procedure. In addition, the difference in the cutoff value for desaturation could be one of the reasons. In our institution, we commonly used 
$100 \%$ oxygen during OLV in both anesthetic techniques. However, the NIVATS group had a slightly lower intraoperative SpO2 than the IVATS group, without desaturation event. This finding indicated that oxygen administration via facemask can prevent hypoxia in NIVATS. Moreover, oxygen toxicity should be a cause of concern. Patients with lung cancer after chemotherapy and exposure to high oxygen concentrations may increase the risk of reactive oxygen species-mediated lung injury ${ }^{(16)}$. In addition, a previous report showed a greater degree of lung injury after $3 \mathrm{~h}$ of OLV in hyperoxic animals ${ }^{(17)}$.

Hypercapnia cannot be prevented in prolonged NIVATS. The main cause is paradoxical respiration and hypoventilation from collapsed non-dependent lung. However, $\mathrm{PacO}_{2}$ was more likely to decrease and return to normal immediately after surgery ${ }^{(18)}$. The peak $\mathrm{ETCO}_{2}$ of the NIVATS group was significantly higher than that of the IVATS group even after the regression analysis. This finding was similar with that of other reports ${ }^{(11,12)}$. The effect of brain metabolism and function in hypercapnia state was controversial, and some studies showed that acute hypercapnia increases intracranial pressure, decreases cerebral perfusion, and precipitates cerebral ischemia ${ }^{(19,20)}$. Another study showed that hypercapnia caused reduced metabolism and spontaneous neural activity in the brain, thereby resulting in a lower arousal state ${ }^{(21)}$. By contrast, several studies revealed that hypercapnia had a neuroprotective effect. Cheng and coworkers assessed patients who experienced hypercapnia during bronchoscopic intervention, and results showed that mild to moderate hypercapnia promoted cognitive activity ${ }^{(22)}$. In addition, permissive hypercapnia is not likely to affect the performances of patients ${ }^{(23)}$.

Other results showed that the overall anesthetic induction and operative times did not differ between the NIVATS and IVATS groups. However, the values in this study were still lower than those in others ${ }^{12,24)}$. These findings might be attributed to the non-routine use of fiberoptic bronchoscopy to validate DLT position and the difference in surgical procedure.

Sore throat is a cause of concern as it affects patient satisfaction after anesthesia. A previous study showed that about $10-43.3 \%$ of patients who were intubated using the double-lumen tube up to $24 \mathrm{~h}$ after surgery presented with sore throat ${ }^{(25)}$. Another research revealed that the incidence of sore throat was high at $57.5 \%$ even in single-lumen intubation ${ }^{(26)}$. Our study showed that such an event was significantly correlated with the intubation technique.

In the era of Enhance Recovery After Surgery (ERAS) program, the non-intubated technique could promote ERAS program based on our study. The NIVATS group had a lower volume of intraoperative blood loss and a shorter length of hospital stay than the IVATS group. Moreover, none of the patients in the NIVATS group presented with sore throat. In terms of other anesthetic complications, the NIVATS group had a higher proportion of patients who experienced nausea and vomiting caused by anesthesia than the IVATS group. However, the results did not significantly differ. Thus, prophylactic treatment for postoperative nausea and vomiting should be included in the standard protocol. 
In our study, the incidence of atelectasis confirmed on chest radiography was low. However, this finding was in contrast with that of the study of Lan and coworkers, and the difference may be associated with the type of surgery. The current research included different types of pulmonary resection procedures, and other studies focused on lobectomy alone.

Currently, NIVATS is used in our institution, and it is applied in several types of VATS procedures ${ }^{(27-29)}$. Therefore, this study could show the benefit of NIVATS particularly in terms of anesthetic perspective because all procedures were performed by a single surgeon with a similar technique.

The current study had several limitations. First, this was a retrospective study. Thus, selection bias could not be totally ruled out. However, bias was equalized via propensity score matching. Second, SpO2 and $\mathrm{ETCO}_{2}$ were not the best parameters that can represent pulmonary gas exchange during OLV. However, noninvasive continuous monitoring was practical during anesthesia, and arterial blood gas analysis might be appropriate in such a case. Third, hemodynamic parameters including time to recovery from anesthesia, pain score, and cognitive function were not considered. Nevertheless, further prospective clinical trials should be conducted to address these limitations.

\section{Conclusions}

IVATS and NIVATS had comparable anesthetic and surgical outcomes. Hence, NIVATS can be alternative technique as it is safe for some patients.

\section{List Of Abbreviations}

NIVATS, non-intubated video-assisted thoracoscopic surgery

IVATS, intubation and controlled ventilation for video-assisted thoracoscopic surgery

OLV, one-lung ventilation

ETco2, end-tidal carbon dioxide

DLT, double-lumen endobronchial tube

Sp02, oxygen saturation

\section{Declarations}

- Declarations; no conflict of interest; this study was approved by the Vajira ethics committee (COA 103/63) of the institution, and it was registered in the Thai Clinical Trials Registry (TCTR20201027004).

- Ethic accordance; all authors confirmed that all method were performed in accordance with the relevant guidelines and regulations. 
- Consent for publication; not applicable

- Author contribution;

Chompunoot Pathonsamit contribute conception, design of the work and write a manuscript Apichat Tantraworasin contribute statistical analysis and write a manuscript

Sujaree Poopipatpab contribute concept and design of work

Sira laohathai contribute conception, design of the work and write a manuscript

- Availability of data and materials; please contact Sira Laohathai. Emai;I sira_l@hotmail.com

- Competing interests

The authors declare that they have no conflict of interest.

- Acknowledgements

We wish to thank all participants especially Unyaparch Rungsidhavaspong for provide data. This study was supported by the Navamindradhiraj University Research Fund.

\section{References}

1. Fitzmaurice BG, Brodsky JB. Airway rupture from double-lumen tubes. J Cardiothorac Vasc Anesth. 1999;13(3):322-9.

2. Miñambres E, Burón J, Ballesteros MA, Llorca J, Muñoz P, González-Castro A. Tracheal rupture after endotracheal intubation: a literature systematic review. Eur J Cardiothorac Surg. 2009;35(6):1056-62.

3. Lim H, Kim JH, Kim D, Lee J, Son JS, Kim DC, et al. Tracheal rupture after endotracheal intubation - A report of three cases. Korean J Anesthesiol. 2012;62(3):277-80.

4. Tan Y, Loganathan N, Thinn KK, Liu EHC, Loh N-HW. Dental injury in anaesthesia: a tertiary hospital's experience. BMC Anesthesiology. 2018;18(1):108.

5. Lohser J, Slinger P. Lung Injury After One-Lung Ventilation: A Review of the Pathophysiologic Mechanisms Affecting the Ventilated and the Collapsed Lung. Anesth Analg. 2015;121(2):302-18.

6. Pompeo E, Tacconi F, Mineo D, Mineo TC. The role of awake video-assisted thoracoscopic surgery in spontaneous pneumothorax. J Thorac Cardiovasc Surg. 2007;133(3):786-90.

7. Pompeo E, Mineo D, Rogliani P, Sabato AF, Mineo TC. Feasibility and results of awake thoracoscopic resection of solitary pulmonary nodules. Ann Thorac Surg. 2004;78(5):1761-8.

8. Pompeo E, Rogliani P, Tacconi F, Dauri M, Saltini C, Novelli G, et al. Randomized comparison of awake nonresectional versus nonawake resectional lung volume reduction surgery. J Thorac Cardiovasc Surg. 2012;143(1):47-54, .e1.

9. Chen JS, Cheng YJ, Hung MH, Tseng YD, Chen KC, Lee YC. Nonintubated thoracoscopic lobectomy for lung cancer. Ann Surg. 2011;254(6):1038-43. 
10. Guo Z, Shao W, Yin W, Chen H, Zhang X, Dong Q, et al. Analysis of feasibility and safety of complete video-assisted thoracoscopic resection of anatomic pulmonary segments under non-intubated anesthesia. J Thorac Dis. 2014;6(1):37-44.

11. Wu CY, Chen JS, Lin YS, Tsai TM, Hung MH, Chan KC, et al. Feasibility and safety of nonintubated thoracoscopic lobectomy for geriatric lung cancer patients. Ann Thorac Surg. 2013;95(2):405-11.

12. Lan L, Cen Y, Zhang C, Qiu Y, Ouyang B. A Propensity Score-Matched Analysis for Non-Intubated Thoracic Surgery. Med Sci Monit. 2018;24:8081-7.

13. Schwarzkopf K, Klein U, Schreiber T, Preussetaler NP, Bloos F, Helfritsch H, et al. Oxygenation during one-lung ventilation: the effects of inhaled nitric oxide and increasing levels of inspired fraction of oxygen. Anesth Analg. 2001;92(4):842-7.

14. Langiano N, Fiorelli S, Deana C, Baroselli A, Bignami EG, Matellon C, et al. Airway management in anesthesia for thoracic surgery: a "real life" observational study. Journal of thoracic disease. 2019;11(8):3257-69.

15. Walsh AM, Lohser J. Arterial Oxygenation and Management of Hypoxemia During VATS. Current Anesthesiology Reports. 2014;4(2):170-6.

16. Grocott HP. Oxygen toxicity during one-lung ventilation: is it time to re-evaluate our practice? Anesthesiol Clin. 2008;26(2):273-80, v.

17. Olivant Fisher A, Husain K, Wolfson MR, Hubert TL, Rodriguez E, Shaffer TH, et al. Hyperoxia during one lung ventilation: inflammatory and oxidative responses. Pediatr Pulmonol. 2012;47(10):979-86.

18. Dong Q, Liang L, Li Y, Liu J, Yin W, Chen H, et al. Anesthesia with nontracheal intubation in thoracic surgery. Journal of thoracic disease. 2012;4(2):126-30.

19. Marshall O, Chawla S, Lu H, Pape L, Ge Y. Cerebral blood flow modulation insufficiency in brain networks in multiple sclerosis: A hypercapnia MRI study. J Cereb Blood Flow Metab. 2016;36(12):2087-95.

20. Zhou Q, Cao B, Niu L, Cui X, Yu H, Liu J, et al. Effects of permissive hypercapnia on transient global cerebral ischemia-reperfusion injury in rats. Anesthesiology. 2010;112(2):288-97.

21. Xu F, Uh J, Brier MR, Hart J, Jr., Yezhuvath US, Gu H, et al. The influence of carbon dioxide on brain activity and metabolism in conscious humans. Journal of cerebral blood flow and metabolism: official journal of the International Society of Cerebral Blood Flow and Metabolism. 2011;31(1):5867.

22. Cheng Q, Li L, Lin D, Li R, Yue Y, Wei H, et al. Effects of acute hypercapnia on cognitive function in patients undergoing bronchoscope intervention. Journal of thoracic disease. 2019;11(3):1065-71.

23. Cheng Q, Zhang J, Wang H, Zhang R, Yue Y, Li L. Effect of Acute Hypercapnia on Outcomes and Predictive Risk Factors for Complications among Patients Receiving Bronchoscopic Interventions under General Anesthesia. PLoS One. 2015;10(7):e0130771.

24. AlGhamdi ZM, Lynhiavu L, Moon YK, Moon MH, Ahn S, Kim Y, et al. Comparison of non-intubated versus intubated video-assisted thoracoscopic lobectomy for lung cancer. $J$ Thorac Dis. 2018;10(7):4236-43. 
25. Jeon J, Lee K, Ahn G, Lee J, Hwang W. Comparison of postoperative sore throat and hoarseness between two types of double-lumen endobronchial tubes: a randomized controlled trial. J Cardiothorac Vasc Anesth. 2015;29(1):121-5.

26. Lee JY, Sim WS, Kim ES, Lee SM, Kim DK, Na YR, et al. Incidence and risk factors of postoperative sore throat after endotracheal intubation in Korean patients. J Int Med Res. 2017;45(2):744-52.

27. Pathonsamit C LS. Non-intubated Uniportal Video-Assisted Thoracoscopic Surgery (NIVATS): First Case Report in Thailand. J Med Assoc Thai. 2019;102:125.

28. Laohathai S, Wannadilok P, Poopipatpab S, Pathonsamit C. Nonintubated Video-Assisted Thoracoscopic Surgery Using Local Anesthesia for Catamenial Pneumothorax. Ann Thorac Surg. 2021;111(4):e245-e6.

29. Laohathai S, Pathonsamit C, Isaranimitkul D, Wannadilok P, Poopipatpab S, Weerayutwattana R. Non-intubated video-assisted thoracoscopic surgery for pulmonary resection in children. Journal of Pediatric Surgery Case Reports. 2021;74:102015. 